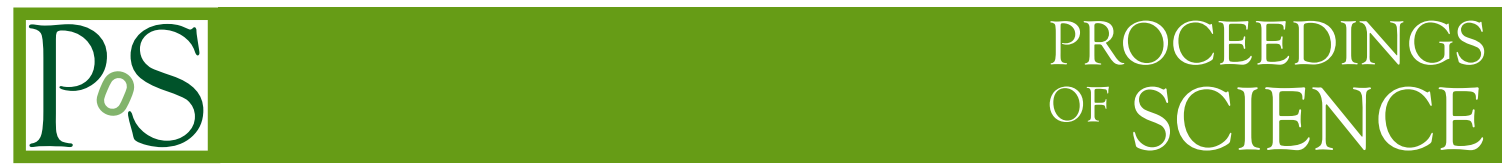

\title{
Di-boson production and limits on triple gauge boson couplings at the Tevatron
}

\section{Thomas J. Phillips*}

Duke University

E-mail: thomas.phillipseduke.edu

The CDF and D0 experiments have observed all varieties of diboson events at the Fermilab Tevatron. These observations are all in statistical agreement with Standard Model expectations, and limits have been placed on non-Standard-Model anamalous triple gauge couplings. New limits from $\mathrm{CDF}$ on $Z \gamma$ anomalous couplings are at least twice as stringent as previously reported couplings.

35th International Conference of High Energy Physics - ICHEP2010,

July 22-28, 2010

Paris France

* Speaker.

${ }^{\dagger}$ For the CDF and D0 Collaborations. 


\section{Introduction}

The structure of self couplings between gauge bosons in the Standard Model (SM) is a direct consequence of electroweak symmetry breaking, so studying these couplings not only confirms the structure of the electroweak sector of the SM, but also presents an opportunity to search for beyondthe-Standard-Model interactions. While direct couplings exist in the SM between $W$ bosons and photons, and between $W$ and $Z$ bosons, there are no Standard Model couplings between the $Z$ boson and the photon, or between the $Z$ boson and itself. Another way to say this is that in the Standard Model, a $W$ boson can radiate a photon or a $Z$ boson, but neither a photon nor a $Z$ boson can radiate either itself or the other. This means that the SM only includes $t$-channel production of $Z \gamma$ and $Z Z$ diboson final states, while $W \gamma, W W$, and $W Z$ diboson final states can be produced by both s- and t-channel processes at tree level. In this paper we examine diboson production in 1.96 $\mathrm{TeV} p \bar{p}$ collisions using the CDF and D0 detectors, compare this production to Standard Model predictions, and set limits on the strength of some anomalous couplings.

\section{2. $W \gamma$ Production}

The Standard Model does have a coupling between the $W$ boson and the photon, but the SM form of this coupling leads to an unusual feature. Destructive interference between diagrams leads to a "radiation amplitude zero" (RAD): no radiation is emitted in a particular direction. This RAD was first predicted in 1979 [2], and it was observed by the D0 collaboration in 2008 [3]. In this publication the D0 collaboration also put limits on non-SM $W \gamma$ couplings, parameterized by $\kappa_{\gamma}$ and $\lambda_{\gamma}$, which are 1 and 0 respectively in the SM. These $95 \%$ C.L. limits are $0.49<\kappa_{\gamma}<1.51$ and $-0.12<\lambda_{\gamma}<0.13$.

\section{3. $Z \gamma$ Production}

Since there is no direct $Z \gamma$ coupling in the Standard Model, the only tree-level diagram for a $Z \gamma$ final state is from initial-state radiation (ISR), where a photon is radiated off an incoming quark. If the $Z$ boson decays into two charged leptons (electrons or muons), then the dilepton mass will generally be near the $Z$-boson mass for ISR. In addition, when the $Z$ decays to charged leptons, it is possible to get radiation off one of these final-state leptons (FSR for final-state radiation). In the case of FSR, the three-body mass $M_{l l \gamma}$ will generally be near the $Z$-boson mass. Were there an anomalous coupling between the photon and the $Z$ boson, it would be possible for a $Z$ to radiate a photon or for a photon to radiate a $Z$, and in this case the two leptons from the $Z$ decay would have a mass near the $Z$ mass, similar to ISR. In particular, anomalous couplings would lead to an increase in the number of $Z \gamma$ events with large $E_{T}^{\gamma}$.

The CDF and D0 collaborations have used two methods to look for $Z \gamma$ events. If the $Z$ boson decays into electrons or muons, it is possible to find $Z \gamma$ events with low backgrounds by first identifying the $Z$ decay and then looking for the additional photon. On the other hand, if the $Z$ boson decays into a pair of neutrinos, these will exit the detector without being seen, so we only see the photon and an imbalance of transverse energy. This signature has significant backgrounds, and these backgrounds are overwhelming at low $E_{T}^{\gamma}$, so the searches for $Z \gamma \rightarrow v v \gamma$ requires a larger photon energy than the searches where the $Z$ boson decays to a pair of charged leptons. 

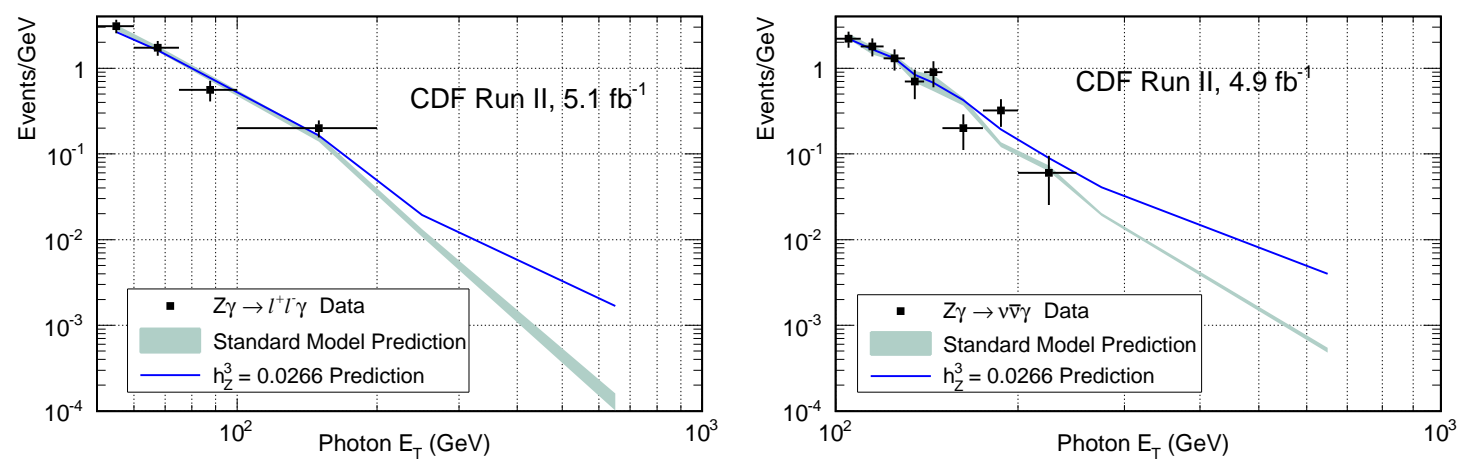

Figure 1: Photon $E_{T}$ distribution in the $Z \rightarrow \mu \mu+Z \rightarrow e e$ datasets for photons with $E_{T}>50 \mathrm{GeV}$ (left). Photon $E_{T}$ distribution in the $Z \rightarrow v v$ dataset for photons with $E_{T}>100 \mathrm{GeV}$ (right).

\begin{tabular}{cccc}
\hline \hline Parameter & $\mathrm{CDF}(\Lambda=1.2 \mathrm{TeV})$ & $\mathrm{CDF}(\Lambda=1.5 \mathrm{TeV})$ & $\mathrm{D} 0(\Lambda=1.5 \mathrm{TeV})$ \\
\hline$h_{3}^{Z}$ & $-0.018,0.020$ & $-0.017,0.016$ & $<0.033$ \\
$h_{4}^{Z}$ & $-0.0009,0.0009$ & $-0.0006,0.0005$ & $<0.0017$ \\
\hline$h_{3}^{\gamma}$ & $-0.022,0.021$ & $-0.017,0.016$ & $<0.033$ \\
$h_{4}^{\gamma}$ & $-0.0009,0.0010$ & $-0.0006,0.0006$ & $<0.0017$ \\
\hline \hline
\end{tabular}

Table 1: Upper limits (95\% C.L.) on anomalous $Z \gamma$ couplings using notation from reference [4] for $\Lambda=1.2$ and $1.5 \mathrm{TeV}$. The D0 limits are from reference [5]; the CDF limits are new.

The most recent Tevatron $Z \gamma$ analysis, using $5 \mathrm{fb}^{-1}$ from CDF, requires $E_{T}^{\gamma}>50 \mathrm{GeV}$ for $Z \rightarrow e^{+} e^{-}$and $Z \rightarrow \mu^{+} \mu^{-}$, and $E_{T}^{\gamma}>100 \mathrm{GeV}$ for $Z \rightarrow v v$. The $E_{T}^{\gamma}>50 \mathrm{GeV}$ requirement eliminate most of the FSR events. The distributions of $E_{T}^{\gamma}$ for these two cases are shown in Figure 1 along with the SM expectations. There is no evidence for an excess at high $E_{T}^{\gamma}$, so limits were set on the anomalous coupling parameters $h_{3}^{Z}, h_{4}^{Z}, h_{3}^{\gamma}$, and $h_{4}^{\gamma}$ (see reference [4] for an explanation of this notation). These limits are shown in Table 1 along with the published limits from the D0 collaboration. An important note is that a cutoff $(\Lambda)$ is required when making the SM predictions in order to avoid unitarity violations, and the limits are a function of this cutoff. Previous results have been published with cutoffs of $\Lambda=1.2 \mathrm{TeV}$ and $\Lambda=1.5 \mathrm{TeV}$, so the new results are reported using both of these values.

There are significant correlations between the $h_{3}$ and $h_{4}$ parameters. Figure 2 shows the twodimensional limits for the new CDF analysis when both parameters are varied at the same time.

\section{4. $W Z$ Production}

The Standard Model includes a $W W Z$ coupling, but it is possible to have anomalous $W W Z$ couplings with a different form, and both collaborations have searched for these anomalous triple gauge couplings. Candidate events are found by searching for three charged leptons and missing transverse energy, where two oppositely charged leptons of the same flavor have a mass consistent with coming from a $Z$ boson, and the third lepton has a transverse mass when combined with the 

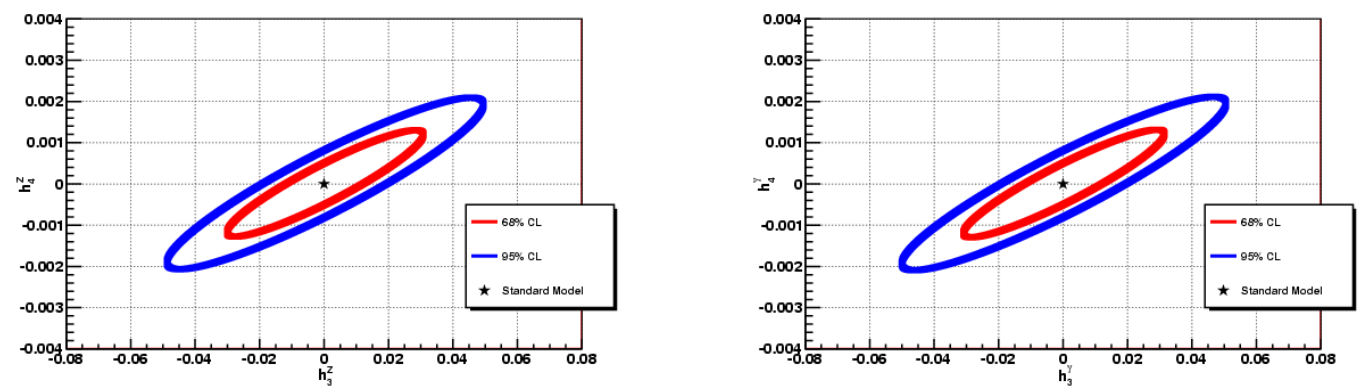

Figure 2: Limits on $h_{3}^{Z}$ vs. $h_{4}^{Z}$ showing significant correlation between the two (left). Limits on $h_{3}^{\gamma}$ vs. $h_{4}^{\gamma}$ also showing significant correlation (right). Both plots are for the cutoff parameter $\Lambda=1.5 \mathrm{TeV}$.

\begin{tabular}{ccc}
\hline \hline Coupling relation & $\mathrm{CDF}(\Lambda=2 \mathrm{TeV})$ & $\mathrm{D} 0(\Lambda=2 \mathrm{TeV})$ \\
\hline$\Delta g_{1}^{Z}=\Delta \kappa_{Z}=0$ & $-0.13<\lambda_{Z}<0.14$ & $-0.075<\lambda_{Z}<0.093$ \\
$\lambda_{Z}=\Delta \kappa_{Z}=0$ & $-0.13<\Delta g_{1}^{Z}<0.23$ & $-0.053<\Delta g_{1}^{Z}<0.156$ \\
$\lambda_{Z}=\Delta g_{1}^{Z}=0$ & $-0.68<\Delta \kappa_{Z}<1.00$ & $-0.376<\Delta \kappa_{Z}<0.686$ \\
\hline \hline
\end{tabular}

Table 2: Limits (95\% C.L.) on anomalous $W Z$ couplings using notation from reference [6] for form factor $\Lambda=2 \mathrm{TeV}$. The CDF limits use $1.9 \mathrm{fb}^{-1}$ of data; the D0 limits use $4.1 \mathrm{fb}^{-1}$ [7].

missing transverse energy consistent with coming from a $W$ boson. Limits on anomalous triple gauge couplings are listed in Table 2.

The cross section for $W Z$ production has been measured by both collaborations. The D0 collaboration has recently reported observing $34 \mathrm{WZ}$ candidate events with an estimated background of $6.0 \pm 0.4$ events in $4.1 \mathrm{fb}^{-1}$ of data for a measured cross section $\sigma(p \bar{p} \rightarrow W Z)=\left(3.90_{-0.90}^{+1.06}\right)$ $\mathrm{pb}$ [7]. The CDF collaboration used $6 \mathrm{fb}^{-1}$ of data to find $50 \mathrm{WZ} \rightarrow 3 l+v$ candidate events with $11.2 \pm 1.6$ expected background and to measure the $W Z$ production cross section to be $\sigma(p \bar{p} \rightarrow$ $W Z)=(4.1 \pm 0.7) \mathrm{pb}$ (preliminary). In a separate measurement CDF used $5.9 \mathrm{fb}^{-1}$ of data to measure $\sigma(p \bar{p} \rightarrow W Z)=(3.7 \pm 0.8) \mathrm{pb}$ (preliminary).

\section{ZZ Production}

The $Z Z$ cross section is small, and when this is combined with the small branching fraction for the $Z$ to decay to charged leptons, very few events are expected for $Z Z \rightarrow e e e e, Z Z \rightarrow e e \mu \mu$, or $Z Z \rightarrow \mu \mu \mu \mu$. Anomalous couplings can produce additional $Z Z$ events, so both collaborations have searched for $Z Z$ events, and when no excess over SM expectations were found, both collaborations set limits on anomalous couplings. These limits, based upon $1 \mathrm{fb}^{-1}$ of data for D0 and $1.9 \mathrm{fb}^{-1}$ of data for CDF are shown in Table 3.

More recent analyses by both collaborations use more data. The D0 collaboration used 1.7 $\mathrm{fb}^{-1}$ of data to report [10] the first $Z Z$ observation with a significance exceeding 5 standard deviations. The CDF collaboration has used $6 \mathrm{fb}^{-1}$ of data to measure the production cross section 


\begin{tabular}{ccc}
\hline \hline Parameter & $\mathrm{CDF}(\Lambda=1.2 \mathrm{TeV})$ & $\mathrm{D} 0(\Lambda=1.2 \mathrm{TeV})$ \\
\hline$f_{4}^{\gamma}$ & $-0.1,0.1$ & $-0.26,0.26$ \\
$f_{4}^{Z}$ & $-0.12,0.12$ & $-0.28,0.28$ \\
\hline$f_{5}^{\gamma}$ & $-0.11,0.11$ & $-0.30,0.28$ \\
$f_{5}^{Z}$ & $-0.13,0.12$ & $-0.31,0.29$ \\
\hline \hline
\end{tabular}

Table 3: Limits (95\% C.L.) on anomalous $Z Z$ couplings using notation from reference [8] for $\Lambda=1.2$. The D0 limits, using $1 \mathrm{fb}^{-1}$, are from reference [9]; the CDF limits use $1.9 \mathrm{fb}^{-1}$.

$\sigma(p \bar{p} \rightarrow Z Z)=\left(1.7_{-0.7}^{+1.2}(\right.$ stat $) \pm 0.2($ syst $\left.)\right) \mathrm{pb}$ (preliminary). For this analysis, $4 \mathrm{ZZ} \rightarrow 4 l$ candidates were observed with $Z Z$ mass below $300 \mathrm{GeV}$. The analysis remains blind to candidate events with larger mass with the intent of searching for heavy resonances that decay into a pair of $Z$ bosons. Neither collaboration used these larger datasets to set limits on anomalous couplings.

\section{Conclusions}

All varieties of diboson events have been observed at the Tevatron. Production rates and kinematic distributions match Standard Model expectations, and limits have been set on anomalous triple-gauge-coupling parameters.

\section{References}

[1] Throughout this paper the notation " $Z$ " is used to specify $Z / \gamma^{*}$ production via the Drell-Yan process. S.D. Drell and T.-M. Yan, Phys. Rev. Lett. 25, 316 (1970).

[2] K.O. Mikaelian, M.A. Samuel, and D. Sahdev, Phys. Rev. Lett. 43, 746 (1979).

[3] V. M. Abazov et al. (D0 Collaboration), Phys. Rev. Lett. 100, 241805 (2008).

[4] U. Baur and E.L. Berger, Phys. Rev. D 47, 4889 (1993).

[5] V.M. Abazov et al. (D0 Collaboration), Phys. Rev. Lett. 102, 201802 (2009).

[6] K. Hagiwara, R.D. Peccei, and D. Zeppenfeld, Phys. Rev. B 282, 253 (1987). K. Hagiwara, J. Woodside, and D. Zeppenfeld, Phys. Rev. D 41, 2113 (1990).

[7] V.M. Abazov et al. (D0 Collaboration), accepted for publication inPhys. Lett. B (2010).

[8] G.J. Gounaris, J. Layssac, and F.M. Renard Phys. Rev. D 62, 073012 (2000).

[9] V.M. Abazov et al. (D0 Collaboration), Phys. Rev. Lett. 100, 131801 (2008).

[10] V.M. Abazov et al. (D0 Collaboration), Phys. Rev. Lett. 101, 171803 (2008). 